\title{
FINDING ANY GIVEN 2-FACTOR IN SPARSE PSEUDORANDOM GRAPHS EFFICIENTLY
}

\author{
JIE HAN, YOSHIHARU KOHAYAKAWA, PATRICK MORRIS, AND YURY PERSON
}

\begin{abstract}
Given an $n$-vertex pseudorandom graph $G$ and an $n$-vertex graph $H$ with maximum degree at most two, we wish to find a copy of $H$ in $G$, i.e. an embedding $\varphi: V(H) \rightarrow V(G)$ so that $\varphi(u) \varphi(v) \in E(G)$ for all $u v \in E(H)$. Particular instances of this problem include finding a triangle-factor and finding a Hamilton cycle in $G$. Here, we provide a deterministic polynomial time algorithm that finds a given $H$ in any suitably pseudorandom graph $G$. The pseudorandom graphs we consider are $(p, \lambda)$-bijumbled graphs of minimum degree which is a constant proportion of the average degree, i.e. $\Omega(p n)$. A $(p, \lambda)$-bijumbled graph is characterised through the discrepancy property: $|e(A, B)-p| A|| B||<\lambda \sqrt{|A||B|}$ for any two sets of vertices $A$ and $B$. Our condition $\lambda=O\left(p^{2} n / \log n\right)$ on bijumbledness is within a $\log$ factor from being tight and provides a positive answer to a recent question of Nenadov.

We combine novel variants of the absorption-reservoir method, a powerful tool from extremal graph theory and random graphs. Our approach is based on that of Nenadov (Bulletin of the London Mathematical Society, to appear) and on ours (arXiv:1806.01676), together with additional ideas and simplifications.
\end{abstract}

\section{INTRODUCTION}

A pseudorandom graph of edge density $p$ is a deterministic graph which shares typical properties of the corresponding random graph $G(n, p)$. These objects have attracted considerable attention in computer science and mathematics. Thomason $[45,46]$ was the first to introduce a quantitative notion of a pseudorandom graph by defining so-called $(p, \lambda)$-jumbled graphs $G$ which satisfy $\left|e(U)-p\left(\begin{array}{c}|U| \\ 2\end{array}\right)\right| \leq \lambda|U|$ for every vertex subset $U \subseteq V(G)$. Ever since, there has been a great deal of investigation into the properties of pseudorandom graphs and this is still a very active area of modern research.

The most widely studied class of jumbled graphs are the so-called $(n, d, \lambda)$-graphs, which were introduced by Alon in the 80s. These graphs have $n$ vertices, are $d$-regular and their second largest eigenvalue in absolute value is at most $\lambda$. An $(n, d, \lambda)$-graph satisfies the expander mixing lemma [8] allowing good control of the edges between any two sets of vertices $A$ and $B$ :

$$
\left|e(A, B)-\frac{d}{n}\right| A|| B||<\lambda \sqrt{|A||B|}
$$

Date: 2019/02/19, 2:09am.

JH was supported by FAPESP (2014/18641-5, 2013/03447-6). YK was partially supported by FAPESP (2013/034476) and CNPq (310974/2013-5, 311412/2018-1, 423833/2018-9). PM is supported by a Leverhulme Trust Study Abroad Studentship (SAS-2017-052\9). YP is supported by the Carl Zeiss Foundation. The cooperation of the authors was supported by a joint CAPES-DAAD PROBRAL project (Proj. no. 430/15, 57350402, 57391197). FAPESP is the São Paulo Research Foundation. CNPq is the National Council for Scientific and Technological Development of Brazil. 
where $e(A, B)=e_{G}(A, B)$ denotes the number of pairs ${ }^{1}(a, b) \in A \times B$ so that $a b$ is an edge of $G$. An illuminating survey of Krivelevich and Sudakov [34] provides a wealth of applications.

There are three interesting regimes in the study of pseudorandom graphs and the class of $(n, d, \lambda)$ graphs is versatile enough to capture the essence of all of these regimes. In the first, one assumes $\lambda=$ $\varepsilon n$, where $n$ is the number of vertices in a graph $G$ and $\varepsilon>0$ is an arbitrary fixed parameter. In this regime one can control edges between sets of linear sizes. This is tightly connected to the theory of quasirandom graphs [15] and the applications of the regularity lemma of Szemerédi [31]. The second regime is when $d$ is constant and $\lambda<d$. This class then contains (non-bipartite) expanders [26] and Ramanujan graphs [38], which are prominent objects of study throughout mathematics and computer science. The third regime (sparse graphs) concerns $\lambda$ being $o(n)$, often some power of $n$, where one has better control on the distribution of edges between truly smaller sets. This case has been investigated more recently and made amenable to some tools from extremal combinatorics.

The focus of this paper will be on conditions under which certain spanning or almost spanning structures are forced in sparse pseudorandom graphs. Our main motivation comes from probabilistic and extremal combinatorics, in particular the problem of universality. A graph $G$ is called $\mathcal{F}$ universal for some family $\mathcal{F}$ if any member $F \in \mathcal{F}$ can be embedded into $G$. This problem attracted a lot of attention [4-7], especially for the case where $\mathcal{F}$ is a class of bounded degree spanning subgraphs. In this case we say an $n$-vertex graph $G$ is $\Delta$-universal if it contains all graphs on at most $n$ vertices of maximum degree $\Delta$. A large part of the focus of the study has been on the universality properties of $G(n, p)[7,18,20-22,27]$. It is also natural to investigate the universality properties of $(n, d, \lambda)$-graphs as was suggested by Krivelevich, Sudakov and Szabó in [35]. In this setting of sparse pseudorandom graphs, a general result on universality has been proved only recently in [1]. Let us comment that the case of dense graphs is well understood since the blowup lemma of Komlós, Sárközy and Szemerédi [29] establishes that pseudorandom graphs of linear minimum degree contain any given bounded degree spanning structure. A little later, the second and fourth author established jointly with Allen, Böttcher and Hàn in [1], a variant of a blow-up lemma for regular subgraphs of pseudorandom graphs. This provides a machinery, complementing the results of Conlon, Fox and Zhao [19] and allowing to transfer many results about dense graphs to sparse graphs in a unified way. However, these results are very general and thus do not establish tight conditions for special cases of spanning structures.

Much more is known for questions about finding one particular spanning structure in a pseudorandom graph and the most prominent spanning structures which were considered in the last fifteen years include perfect matchings, studied by Alon, Krivelevich and Sudakov in [34], Hamilton cycles studied by Krivelevich and Sudakov [33], clique-factors [23, 24,35,41] and powers of Hamilton cycles [3].

The problem of when a triangle-factor ${ }^{2}$ appears in a given $(n, d, \lambda)$-graph has been a prominent question and is an instructive insight into the behaviour of pseudorandom graphs. It is easy to infer from the expander mixing lemma that if $\lambda \leq 0.1 d^{2} / n$, then any $(n, d, \lambda)$-graph contains a triangle

\footnotetext{
$\overline{1_{\text {Note that edges }}}$ in $A \cap B$ are counted twice.

${ }^{2}$ That is, disjoint copies of $K_{3}$ covering all the vertices.
} 
(in fact, every vertex lies in a triangle). An ingenious construction of Alon [9] provides an example of a triangle-free $(n, d, \lambda)$-graph with $\lambda=\Theta\left(n^{1 / 3}\right)$ and $d=\Theta\left(n^{2 / 3}\right)$, which is essentially as dense as possible, considering the previous comments. This example can be bootstrapped, as is done in [35], to the whole possible range of $d=d(n)$, giving $K_{3}$-free $(n, d, \lambda)$-graphs with $\lambda=\Theta\left(d^{2} / n\right)$. Further examples of (near) optimal dense pseudorandom triangle-free graphs have since been given $[17,32]$. On the other hand, Krivelevich, Sudakov and Szabó [35] proved that $(n, d, \lambda)$-graphs with $\lambda=o\left(d^{3} /\left(n^{2} \log n\right)\right)$ contain a triangle-factor if $3 \mid n$ and they made the following intriguing conjecture, which is one of the central problems in the theory of spanning structures in $(n, d, \lambda)$ graphs.

Conjecture 1.1 (Conjecture 7.1 in [35]). There exists an absolute constant $c>0$ such that if $\lambda \leq c d^{2} / n$, then every $(n, d, \lambda)$-graph $G$ on $n \in 3 \mathbb{N}$ vertices has a triangle-factor.

This conjecture is supported by their result that $\lambda \leq 0.1 d^{2} / n$ implies the existence of a fractional triangle-factor. Furthermore, a recent result of three of the authors [23, 25] states that, under the condition $\lambda \leq(1 / 600) d^{2} / n$, any $(n, d, \lambda)$-graph $G$ with $n$ sufficiently large contains a family of vertexdisjoint triangles covering all but at most $n^{647 / 648}$ vertices of $G$, thus a 'near-perfect' triangle-factor. A very recent, remarkable result of Nenadov [41] infers that $\lambda \leq c d^{2} /(n \log n)$ for some constant $c>0$ is sufficient to yield a triangle-factor. Considering the triangle-free constructions mentioned above, we see that Nenadov's result is within a log factor of the optimal conjectured bound. Nenadov also raised the question in [41] of whether a similar condition would imply the existence of any given 2 -factor ${ }^{3}$ in a pseudorandom graph. The purpose of this work is to give a positive answer to Nenadov's question, casting the question in terms of 2-universality and showing that we can efficiently find a given maximum degree 2 subgraph in polynomial time.

In order to state our result we will switch ${ }^{4}$ to working with $(p, \lambda)$-bijumbled graphs (introduced in [28]), which give a convenient, slight variant of Thomason's jumbledness. Bijumbled graphs $G$ satisfy the property:

$$
|e(A, B)-p| A|| B||<\lambda \sqrt{|A||B|}
$$

for all $A, B \subseteq V(G)$. In particular it is easy to see by the expander mixing lemma (1) that an $(n, d, \lambda)$-graph is $(d / n, \lambda)$-(bi)jumbled. Moreover the two concepts are closely linked as a $(p, \lambda)$ (bi)jumbled graph is almost $p n$-regular, in that almost all vertices have degree close to $p n$.

Before the current paper, the best result towards 2-universality in pseudorandom graphs is due to Allen, Böttcher, Hàn and two of the authors [3]. There, they proved that there exists an $\varepsilon>0$ such that $\left(p, \varepsilon p^{5 / 2} n\right)$-bijumbled graphs of minimum degree $\Omega(p n)$ contain a square ${ }^{5}$ of a Hamilton cycle and hence are 2-universal. The proof is algorithmic, leading to an efficient procedure. Here we weaken the requirement on $\lambda$ to match that of Nenadov and obtain the following.

Theorem 1.2. For all $\delta>0$, there exist constants $\varepsilon>0$ and $n_{0}$ such that, for any $p \in(0,1]$, the following holds. For any $n \geq n_{0}$ and any given potential 2-factor $F$ (that is, family of disjoint cycles

\footnotetext{
${ }^{3} \mathrm{~A} 2$-factor is a 2-regular spanning subgraph.

${ }^{4}$ Nenadov also worked in this broader class of pseudorandom graphs.

${ }^{5} \mathrm{~A}$ square of a graph $H$ is obtained by connecting its vertices at distance at most two through edges. The existence of a square of a Hamilton cycle implies 2-universality as one can greedily find vertex-disjoint cycles of arbitrary lengths.
} 
whose lengths sum up to $n)$, there is a polynomial time algorithm which finds a copy of $F$ in any $(p, \lambda)$-bijumbled graph $G$ on $n$ vertices with $\lambda \leq \varepsilon p^{2} n / \log n$ and minimum degree $\delta(G) \geq \delta p n$.

In particular, Theorem 1.2 implies that such a $(p, \lambda)$-bijumbled graph $G$ is 2-universal. Indeed, given a graph $F^{\prime}$ on at most $n$ vertices with $\Delta\left(F^{\prime}\right) \leq 2$, we find a supergraph $F$ of $F^{\prime}$ on $n$ vertices, so that all but at most one of the components of $F$ are cycles. It is possible that $F$ may have either one isolated vertex or a single edge but since we can easily embed a single vertex/edge into a bijumbled graph $G$ altering its minimum degree only a little, it suffices to concentrate on the case that $F$ is a 2 -factor.

1.1. Proof method. Our proof uses the absorption-reservoir method, which has been a powerful tool in proving the existence of certain substructures and is often superior to the aforementioned blow-up lemmas. The basic idea of the method is to set up a flexible absorbing structure which creates an extra 'legroom' when trying to embed the last $o(n)$ vertices. The beginnings of this method date back to the early 90 s, but the breakthrough in the wide applicability of these methods, however, was first established by Rödl, Ruciński and Szemerédi [43,44] in their study of Hamiltonicity in hypergraphs. There, the method was used to study dense hypergraphs but the methods have since been adapted to other settings (see e.g. $[2,3,36]$ ).

In his work on spanning trees in random graphs [40], Montgomery ingeniously wove sparse 'robust' bipartite graphs (which we call sparse templates) into the absorption-reservoir method. The first use of sparse templates for the absorption in the context of pseudorandom graphs was recently given by the current authors in [24]. Our approach here builds on the ideas from our paper [24], and introduces for the first time, an efficient version of this new type of absorption. In order to explicitly generate a sparse template we use bounded degree bipartite graphs with strong expansion properties. Such graphs are known as concentrators $[11,26]$. We also build upon the different absorbing-type argument due to Nenadov [41]. Our approach combines both arguments, making them constructive by replacing certain nonalgorithmic arguments from [41] and derandomising additional arguments at various places.

\section{Proof of Theorem 1.2}

The following three theorems will establish our main result. Note that the non-algorithmic version of Theorem 2.1 was proved in [41].

Theorem 2.1 (Theorem 1.2, [41]). For every $\delta>0$ there exists a constant $\varepsilon>0$ such that, for any $p \in(0,1]$, a $(p, \lambda)$-bijumbled graph $G$ on $n \in 3 \mathbb{N}$ vertices with $\lambda \leq \varepsilon p^{2} n / \log n$ and minimum degree $\delta(G) \geq \delta$ pn contains a triangle-factor, which can be found in polynomial time.

Theorem 2.2. For every $\delta>0$ and $L \in \mathbb{N}$ there exist constants $\varepsilon_{0}=\varepsilon_{0}(\delta, L)>0$ and $n_{0}$ such that for any $0<\varepsilon<\varepsilon_{0}$ the following holds. Let $G$ be a $(p, \lambda)$-bijumbled graph on $n \geq n_{0}$ vertices with

$p \in(0,1 / 2], \lambda \leq \varepsilon p^{2} n$ and minimum degree $\delta(G) \geq \delta p n$. Then in polynomial time, one can find any family of vertex-disjoint cycles with lengths in the interval $[4, L]$ whose lengths sum up to at most $n$. 
Theorem 2.3. For every $\delta>0$ there exist constants $L \in \mathbb{N}, \varepsilon_{1}>0$ and $n_{0}$ such that the following holds. For any $p \in(0,1 / 3]$ and $0<\varepsilon<\varepsilon_{1}$, let $G$ be a $(p, \lambda)$-bijumbled graph on $n \geq n_{0}$ vertices with $\lambda \leq \varepsilon p^{2} n$ and minimum degree $\delta(G) \geq \delta p n$. Then in polynomial time, one can find any family of vertex-disjoint cycles with lengths in the interval $[L+1, n]$ whose lengths sum up to at most $n$.

Now we can quickly derive Theorem 1.2.

Proof of Theorem 1.2. We consider three (not mutually exclusive) cases:

(i) at least $n / 2$ vertices of $F$ are covered by vertex-disjoint triangles,

(ii) at least $n / 4$ vertices of $F$ are covered by vertex-disjoint cycles with lengths in the interval $[4, L]$ where $L$ is some absolute constant determined by Theorem 2.3 above,

(iii) at least $n / 4$ vertices of $F$ are covered by vertex-disjoint cycles with lengths in the interval $[L+1, n]$.

For a given 2-factor $F$ on at most $n$ vertices, we are in one of the three cases defined above. Let $F_{1}, F_{2}$ and $F_{3}$ denote the subgraphs of $F$, so that all triangles constitute $F_{1}$, all cycles with lengths in $[4, L]$ constitute the subfamily $F_{2}$ and all cycles of length at least $L+1$ are $F_{3}$. We set $n_{i}:=v\left(F_{i}\right)$ for each $i \in[3]$.

If we are in the first case $\left(n_{1} \geq n / 2\right)$ we partition the vertex set $V$ of $G$ into three parts $V_{1} \dot{\cup} V_{2} \cup V_{3}$, so that $\left|V_{1}\right|=n / 2$ and $\left|V_{3}\right|=\left|V_{4}\right|=n / 4$ and each $G\left[V_{i}\right]$ remains a $(p, \lambda)$-bijumbled graph. Moreover, every vertex $v \in V$ satisfies $\operatorname{deg}\left(v, V_{i}\right) \geq \delta p\left|V_{i}\right| / 2$ for any $i \in$ [3]. Clearly, one could achieve this via a random partition and it will be possible to derandomise this approach (see Corollary 3.8). If $n_{2} \geq n_{3}$, then we first apply Theorem 2.3 to embed $F_{3}$ via some embedding $\varphi_{3}$ into $G\left[V_{3}\right]$. Then Theorem 2.2 asserts that $F_{2}$ can be embedded into $G_{2}^{\prime}:=G\left[\left(V_{2} \cup V_{3}\right) \backslash \varphi_{3}\left(V\left(F_{3}\right)\right)\right]$, since $G_{2}^{\prime}$ is itself a $(p, \lambda)$-bijumbled graph with minimum degree $\delta p n / 4$. Finally, we apply Theorem 2.1 to embed $F_{1}$ into the remaining graph (which is again $(p, \lambda)$-bijumbled graph with minimum degree at least $\delta p n / 2)$. If $n_{3} \geq n_{2}$ then we first embed $F_{2}$, then $F_{3}$ and, finally, $F_{1}$. The other cases $n_{2} \geq n / 4$ and $n_{3} \geq n / 4$ are treated analogously.

2.1. Structure of the paper. It remains to prove Theorems $2.1-2.3$. We will only consider the case $p \leq 1 / 3$, since the dense case can be treated fairly easily by the algorithmic version of the blow-up lemma due to Komlós, Sárközy and Szemerédi [30]. In Section 3 we collect some notation and useful tools and algorithms for our study. In the subsequent two sections we prove the first two theorems (Theorems 2.2 and 2.3) and in Section 6 we replace one non-algorithmic argument from [41] with a constructive proof.

Throughout we use the shorthand $(p, \lambda)$-graphs to refer to $(p, \lambda)$-bijumbled graphs, we write log to denote the natural logarithm and we omit floor and ceiling signs in order not to clutter the arguments. The final section closes with some problems left for further study.

\section{Auxiliary Results}

3.1. Simple statements about $(p, \lambda)$-bijumbled graphs. Recall that we will often refer to $(p, \lambda)$-bijumbled graphs as $(p, \lambda)$-graphs. In this section, we collect some useful properties of $(p, \lambda)$ graphs. We will use the following notation. Given a graph $G=(V, E)$, we denote by $\operatorname{deg}(v, U)$ the 
number of neighbours of $v \in V$ in $U \subseteq V$. A $u$-v-path is a path $P$ with end vertices $u$ and $v$, and we call the other vertices of $P$ the inner vertices. For vertex subsets $A, B$, an $A$ - $B$ path is a $u-v$ path for some vertices $u \in A$ and $v \in B$. The length of a path is the number of its edges. Finally we will denote by $C_{\ell}(1, \ldots, 1, K)$, the graph that consists of a path $P$ of length $\ell-2$, whose end vertices have exactly $K$ distinct common neighbours outside of $V(P)$, for some $K \in \mathbb{N}$. We start with the following remark which follows directly from the definition (2).

Remark 3.1. If $\varepsilon>0$ and $A$ and $B$ are subsets of $a(p, \lambda)$-graph with $\lambda \leq \varepsilon p^{2} n$, such that $|A \| B| \geq$ $4 \varepsilon^{2} p^{2} n^{2}$, then $e(A, B) \geq \frac{p|A \|| B \mid}{2}$.

Next, we show a well-known property of bijumbled graphs; that they can not be too sparse.

Proposition 3.2. Given $\varepsilon \in(0,1)$, there exists $n_{0} \in \mathbb{N}$ such that if $G=(V, E)$ is a $(p, \lambda)$-graph on $n \geq n_{0}$ vertices with $\lambda \leq \varepsilon p^{2} n$ and $\varepsilon p \leq 1 / 2$, then $p \geq\left(\varepsilon^{2} n\right)^{-1 / 3} / 4$.

Proof. Let $S \subseteq V$ be a set of at least $n / 2$ vertices. Then there is a vertex $v \in S$ whose degree in $G[S]$ is at most $2 p|S|$. Indeed, we have $\sum_{v \in S} \operatorname{deg}(v, S)=2 e(G[S]) \leq p|S|^{2}+\lambda|S|$, which implies that the average degree in $G[S]$ is at most $p|S|+\lambda \leq 2 p|S| \leq 2 p n$.

We consecutively find vertices $v_{1}, \ldots, v_{t}$ with $t=n /(2+4 p n)$ such that setting $V_{i}:=V$ > $\left\{v_{1}, \ldots, v_{i-1}\right\}$, we have $\operatorname{deg}\left(v_{i}, V_{i}\right) \leq 2 p\left|V_{i}\right| \leq 2 p n$. Thus setting $U:=\left\{v_{i}: i \in[t]\right\}$ and $W:=$ $V \backslash\left(U \cup \bigcup_{i \in[t]} N\left(v_{i}\right)\right)$, we have that $|W| \geq n-t(1+2 p n)=n / 2$ and $e_{G}(U, W)=0 \geq p|U||W|-\lambda \sqrt{|U||W|}$.

It follows that $\varepsilon p^{2} n \geq \lambda \geq p \sqrt{t n / 2}$. Thus, $2 \varepsilon^{2} p^{2} n \geq t=n /(2+4 p n)$, which implies $p^{2} \geq$ $\left(\varepsilon^{-2} / 4\right) \min \{1 /(4 p n), 1 / 2\}$. Rearranging we get $p \geq \min \left\{\left(\varepsilon^{2} n\right)^{-1 / 3} / 3,1 /(2 \sqrt{2} \varepsilon)\right\} \geq\left(\varepsilon^{2} n\right)^{-1 / 3} / 4$ for $n$ sufficiently large.

The following fact also concerns the edge distribution of bijumbled graphs.

Fact 3.3. Let $\varepsilon>0$ and $G$ be a $(p, \lambda)$-graph on $n$ vertices with $p \in(0,1]$ and $\lambda \leq \varepsilon p^{2} n$.

(i) If $U$ is a set of vertices, then there are at most $4 \varepsilon^{2} p^{2} n^{2} /|U|$ vertices $w$ in $G$ with $\left|N_{G}(w) \cap U\right|<$ $p|U| / 2$.

(ii) Given an integer $t$ and vertex sets $U_{1}, \ldots, U_{t}, W$ such that $|W|>\sum_{i=1}^{t} 4 \varepsilon^{2} p^{2} n^{2} /\left|U_{i}\right|$, we can find a vertex $w \in W$ such that $\left|N_{G}(w) \cap U_{i}\right| \geq p\left|U_{i}\right| / 2$ for all $i \in[t]$ in time $O(t p n|W|)$.

Proof. Let $U^{\prime}$ be the set of vertices $w$ such that $\left|N_{G}(w) \cap U\right|<p|U| / 2$. From (2) we have $\left|U^{\prime}\right| p|U| / 2>$ $e\left(U^{\prime}, U\right) \geq p|U|\left|U^{\prime}\right|-\lambda \sqrt{|U|\left|U^{\prime}\right|}$. The conclusion follows from rearranging.

By the first part of the fact, $W$ clearly contains a desired vertex. We find it by screening the degree of any vertex of $W$ into each $U_{i}$, which takes time $t \cdot O(p n)$. Since we may fail for at most $|W|$ times, the conclusion follows.

Next, given two sets $A, B$ and $C$, we show how to find an $A$-B-path of given length such that the inner vertices are from $C$.

Proposition 3.4. Let $\varepsilon>0, \ell \in \mathbb{N}$ and $G$ be $a(p, \lambda)$-graph on $n$ vertices with $p \in(0,1], \lambda \leq \varepsilon p^{2} n$ and $\varepsilon p n \geq 1$. If $A$ and $B$ are sets of at least $2^{\ell-1}$ Epn vertices and $C$ is a set of at least $2^{\ell-1} \varepsilon n$ vertices, then we can find an $A$-B-path $P$ of length $\ell$ whose inner vertices lie in $C$ in time $O\left(p^{2} n^{2}\right)$. 
Proof. If $\ell=1$ then we have $e(A, B)>p|A||B|-\lambda \sqrt{|A||B|} \geq \sqrt{|A||B|}(p \varepsilon p n-\lambda) \geq 0$, namely, there is an edge with one end in $A$ and the other in $B$. We can find such an edge by searching vertices one by one in $A$. Since for each vertex it takes time $O(p n)$ and by Fact $3.3(i)$, we may fail for at most $4 \varepsilon p n$ vertices, this can be done in time $O\left(p^{2} n^{2}\right)$. We proceed now inductively and we assume that $\ell \geq 2$ and the assumption holds for $\ell-1$.

By Fact $3.3(i i)$ we find a vertex $a \in A$ with degree at least $p|C| / 2$ into $C$, in time $O\left(p^{2} n^{2}\right)$ (because we could focus the search on a set $W$ of at most $p n$ vertices in $A$ ). Applying our inductive hypothesis to $N(a) \cap C, B \backslash\{a\}$ and $C \backslash\{a\}$ we find an $(N(a) \cap C)-(B \backslash\{a\})$-path of length $\ell-1$ with inner vertices in $C$, which together with $a$ yields the desired path of length $\ell$.

We will use copies of $C_{\ell}(1, \ldots, 1, K)$ in our absorbing structure. The following simple fact asserts that we can find these copies in any large enough set of vertices.

Fact 3.5. Let $\varepsilon>0, K \in \mathbb{N}$ and let $G$ be a $(p, \lambda)$-graph on $n$ vertices with $p \in(0,1]$ and $\lambda \leq \varepsilon p^{2} n$. Let $\varepsilon p^{2} n \geq K / 4, \ell \geq 4$ and $U$ be a set of at least $2^{\ell} \varepsilon n$ vertices. Then we can find a copy of $C_{\ell}(1, \ldots, 1, K)$, and thus also a copy of $C_{\ell}$ in $U$, in time $O\left(p^{2} n^{2}\right)$.

Proof. Let $U_{1}^{\prime}$ be the set of vertices $v \in U$ with $|N(v) \cap U|<p|U| / 2$. Since $|U| \geq 2^{\ell} \varepsilon n$, Fact $3.3(i)$ implies that $\left|U_{1}^{\prime}\right| \leq \varepsilon p^{2} n / 4$. We fix a vertex $u_{1} \in U \backslash U_{1}^{\prime}$, i.e. $\operatorname{deg}\left(u_{1}, U\right) \geq p|U| / 2$. Let $U_{2}^{\prime}$ be the set of vertices $v \in U$ with $\left|N(v) \cap\left(N\left(u_{1}\right) \cap U\right)\right|<p\left|N\left(u_{1}\right) \cap U\right| / 2$. Since $\left|N\left(u_{1}\right) \cap U\right| \geq p|U| / 2 \geq 8 \varepsilon p n$, Fact $3.3(i)$ implies that $\left|U_{2}^{\prime}\right| \leq \varepsilon p n / 2$. Thus, we have $\left|U_{1}^{\prime} \cup U_{2}^{\prime}\right| \leq \varepsilon p n$.

We choose an arbitrary vertex $u_{2} \in U \backslash\left(U_{1}^{\prime} \cup U_{2}^{\prime} \cup\left\{u_{1}\right\}\right)$. If $\ell=4$ then we clearly find a copy of $C_{\ell}(1,1,1, K)$ in $U$, because $\left|N\left(u_{1}\right) \cap N\left(u_{2}\right) \cap U\right| \geq p^{2}|U| / 4 \geq K+1$. If $\ell \geq 5$, then we first set aside a set $W$ of $K$ vertices from the common neighbourhood of $u_{1}$ and $u_{2}$. Now due to the fact that $\left|\left(N\left(u_{i}\right) \cap U\right) \backslash\left(W \cup\left\{u_{1}, u_{2}\right\}\right)\right| \geq 2^{\ell-2} \varepsilon p n$ for $i=1,2$ and $\left|U \backslash\left(W \cup\left\{u_{1}, u_{2}\right\}\right)\right| \geq 2^{\ell-2} \varepsilon n$, we find by Proposition 3.4 a path of length $\ell-4$ between $N\left(u_{1}\right) \cap U$ and $N\left(u_{2}\right) \cap U$, which together with $u_{1}$, $u_{2}$ and $W$, forms a copy of $C_{\ell}(1, \ldots, 1, K)$.

For the running time, by Fact $3.3(i i)$, we can find $u_{1}$ and $u_{2}$ in time $O\left(p^{2} n^{2}\right)$ (because we could focus on a set of at most $p n$ vertices in $U)$. The rest of the proof runs in time $O\left(p^{2} n^{2}\right)$, because we use Proposition 3.4.

The following lemma asserts that we can (greedily) find almost spanning paths in $(p, \lambda)$-graphs.

Lemma 3.6. Let $\varepsilon>0$ and $G$ be $a(p, \lambda)$-graph on $n$ vertices with $p \in(0,1 / 2]$ and $\lambda \leq \varepsilon p^{2} n$. If $U$ is a vertex subset of size greater than $\varepsilon n$, then we can find any path of length $\ell \leq|U|-\varepsilon n$ in $U$ in time $O\left(\ell \cdot p^{2} n^{2}\right)$.

Proof. By Fact 3.3 there is a vertex $u \in U$ of degree at least $p|U| / 2$ in $U$. This gives us a path of length 0. Assume now that we found inductively a path $P_{t}=u_{0} u_{1} \ldots u_{t}$ of length $t \leq\lfloor|U|-\varepsilon n\rfloor-1$ such that $\operatorname{deg}\left(u_{t}, U \backslash V\left(P_{t}\right)\right) \geq p\left|U \backslash V\left(P_{t}\right)\right| / 2$. Then by Fact $3.3(i)$, as $\left|U \backslash V\left(P_{t}\right)\right| \geq \varepsilon n$ and using that $p \leq 1 / 2$, we have that there exists a vertex $u_{t+1} \in N\left(u_{t}\right) \cap\left(U \backslash V\left(P_{t}\right)\right)$ with $\operatorname{deg}\left(u_{t+1}, U \backslash V\left(P_{t}\right)\right) \geq$ $p\left|U \backslash V\left(P_{t}\right)\right| / 2$ and the induction step is complete.

Since the proof is a repeated application of Fact $3.3(i)$, Fact $3.3(i i)$ implies that the running time is $\ell \cdot O\left(p^{2} n^{2}\right)$. 
3.2. Partitioning vertex sets. At various points in our proof, we will wish to partition our vertex set in such a way that every vertex maintains good degree to all parts of the partition. This can be easily achieved probabilistically by choosing a random partition. However this idea can also be derandomised and achieved computationally efficiently. We use the following theorem of Alon and Spencer.

Theorem 3.7 (Theorem 16.1.2 in [13]). Let $\left(a_{i j}\right)_{i, j=1}^{n}$ be an $n \times n$ 0/1-matrix. Then one can find, in polynomial time, $\varepsilon_{1}, \ldots, \varepsilon_{n} \in\{-1,1\}$ such that for every $1 \leq i \leq n$, it holds that $\left|\sum_{j=1}^{n} \varepsilon_{j} a_{i j}\right| \leq$ $\sqrt{2 n \log (2 n)}$.

Corollary 3.8. Let $k \in \mathbb{N} \varepsilon, \beta, \delta>0$ and $p \in(0,1]$. Then there exists $n_{0} \in \mathbb{N}$ such that for any $(p, \lambda)$-graph $G$ on $n \geq n_{0}$ vertices such that $\lambda \leq \varepsilon p^{2} n$, the following holds. Let $U, W \subseteq V(G)$ be subsets of vertices such that $|U| \geq \beta n$ and for all $w \in W, \operatorname{deg}(w, U) \geq \delta p|U|$. Then in polynomial time, we can find $s:=2^{k}$ sets $U_{1}, \ldots, U_{s} \subseteq U$ such that $U=U_{1} \dot{\cup} \ldots \dot{U} U_{s}$, for each $i$ we have $\left|U_{i}\right|=|U| / s$ and for all $w \in W$ and $i \in[s], \operatorname{deg}\left(w, U_{i}\right) \geq \delta p\left|U_{i}\right| / 2$.

Proof. We apply Theorem 3.7 to the adjacency matrix of $G$, where we add an all one row and an extra column and impose that row $i$ is all zero if $i \notin W$ and column $j$ is all zero if $j \notin U$. We let $U_{b}^{\prime}=\left\{j \in U: \varepsilon_{j}=(-1)^{b}\right\}$, for $b=1,2$. The last row of the matrix guarantees that $\left\|U_{1}^{\prime}|-| U_{2}^{\prime}\right\| \leq$ $\sqrt{2(n+1) \log (2 n+1)}=: g(n)$. The other rows guarantee that the vertices in $W$ have good degree to both sets, so that after moving some vertices from one of the sets to another in order to balance $\left|U_{1}^{\prime}\right|$ and $\left|U_{2}^{\prime}\right|$, we have that for all $w \in W, \operatorname{deg}\left(w, U_{i}^{\prime}\right) \geq \delta p|U| / 2-2 g(n)$.

We can now apply the above procedure to each $U_{i}^{\prime}$, with the new minimum degrees. Repeating this $k$ times, we end up with $U_{1}, \ldots, U_{s}$ as an equipartition of $U$ such that for any $w \in W$, $\operatorname{deg}\left(w, U_{i}\right) \geq \delta p|U| / s-2 k g(n)$. Owing to Proposition 3.2, we are done because for sufficiently large $n, 2 k g(n) \leq \delta \beta p n /(2 s) \leq \delta p|U| /(2 s)$.

3.3. A connecting lemma. The lemma below allows us to close many paths (whose ends are 'well-connected' into a large set) into cycles using short paths of a fixed prescribed length. In the following lemma a $v$ - $v$-path refers to a cycle through $v$ whose inner vertices are all the vertices of the cycle not equal to $v$.

Lemma 3.9 (Multiple connection lemma). For every $0<\beta, \delta^{\prime} \leq 1, \ell \geq 3$ there exists $\varepsilon_{0}>0$ and $n_{0} \in \mathbb{N}$ such that for all $\varepsilon \in\left(0, \varepsilon_{0}\right)$ and $n \geq n_{0}$ the following holds. Let $G$ be a $(p, \lambda)$-graph on $n$ vertices with $p \in(0,1]$ and $\lambda \leq \varepsilon p^{2} n$. Let $U$ be a vertex subset of size at least $\beta n$ and $\left(a_{i}, b_{i}\right)_{i \in[r]} a$ system of pairs of vertices in $G$, so that every vertex occurs at most twice in $\left(a_{1}, \ldots, a_{r}, b_{1}, \ldots, b_{r}\right)$ and $U$ is disjoint from $\bigcup_{i}\left\{a_{i}, b_{i}\right\}$. If $r \leq|U| /(8 \ell)$ and $\operatorname{deg}\left(a_{i}, U\right), \operatorname{deg}\left(b_{i}, U\right) \geq \delta^{\prime} p|U|$ for all $i \in[r]$ then the following holds. In polynomial time, we can find a family $\mathcal{Q}$ of length $\ell a_{i}-b_{i}$-paths $Q_{i}$, whose inner vertices are pairwise disjoint and lie in $U$.

Proof. Fix $\varepsilon_{0} \leq \delta^{\prime} \beta 2^{-(\ell+6)} / \ell$. Firstly, using Corollary 3.8, in polynomial time, we can split $U$ into $U=U_{1} \dot{\cup} U_{2}$ such that $\left|U_{1}\right|=\left|U_{2}\right|=|U| / 2$ and $\operatorname{deg}\left(a_{i}, U_{b}\right), \operatorname{deg}\left(b_{i}, U_{b}\right) \geq \delta^{\prime} p|U| / 4$ for all $i$ and $b=1,2$. We will build our paths algorithmically in two phases, first using vertices of $U_{1}$ and then vertices of $U_{2}$. We initiate by letting $\mathcal{Q}^{\prime}=\varnothing, U_{1}^{\prime}=U_{1}$ and $U_{2}^{\prime}=U_{2}$. We will use $\mathcal{Q}^{\prime}$ to denote our intermediate 
family of paths and $U_{1}^{\prime}, U_{2}^{\prime}$ the remaining sets of vertices that we can use. Note that throughout we will have $|V(\mathcal{Q})| \leq r \ell \leq|U| / 8$, and thus $\left|U_{1}^{\prime}\right|,\left|U_{2}^{\prime}\right|$ will always have size at least $|U| / 4$.

We proceed as follows. If there is an $i \in[r]$ such that $\operatorname{deg}\left(a_{i}, U_{1}^{\prime}\right), \operatorname{deg}\left(b_{i}, U_{1}^{\prime}\right) \geq \delta^{\prime} p|U| / 8$, then using Proposition 3.4, in time $O\left(p^{2} n^{2}\right)$ we find a length $\ell-2$ path $P_{i}$ from a vertex in $N\left(a_{i}\right) \cap U_{1}^{\prime}$ to a vertex in $N\left(b_{i}\right) \cap U_{1}^{\prime}$ using vertices in $U_{1}^{\prime}$. Add $Q_{i}:=a_{i}-P_{i}-b_{i}$ to $\mathcal{Q}$ and delete the vertices of $P_{i}$ from $U_{1}^{\prime}$. At the end of this phase, let $I \subseteq[r]$ be the remaining indices. Since each vertex appears at most twice in $\left(a_{i}, b_{i}\right)_{i \in[r]}$, by Fact $3.3(i)$, we have that

$$
|I| \leq \frac{4 \varepsilon^{2} p^{2} n^{2}}{\left|U_{1}^{\prime}\right|} \leq \frac{4 \varepsilon^{2} p^{2} n^{2}}{\beta n / 4} \leq 16 \varepsilon_{0} p^{2} n \leq \delta^{\prime} p^{2}|U| /(8 \ell) \leq \delta^{\prime} p|U| /(8 \ell)
$$

where we used $\left|U_{1}^{\prime}\right| \geq|U| / 4 \geq \beta n / 4$, and $\varepsilon \leq \varepsilon_{0} \leq \delta^{\prime} \beta 2^{-(\ell+6)} / \ell$. Now we run the process again, using $U_{2}$ in place of $U_{1}$. As $\left|V\left(\mathcal{Q}^{\prime}\right) \cap U_{2}\right| \leq \delta^{\prime} p|U| / 8$ throughout, we can proceed greedily by the degree assumptions and complete the family $\mathcal{Q}$. Note that in each step, we need to screen the degrees of the remaining pair $a_{i}$ and $b_{i}$, which can be done in time $O(r p n)$. Then the application of Proposition 3.4 runs in time $O\left(p^{2} n^{2}\right)$. In total the algorithm runs in time $O\left(r p^{2} n^{2}\right)$.

3.4. An explicit template. A template $T$ with flexibility $m \in \mathbb{N}$ is a bipartite graph on $7 m$ vertices with vertex parts $I$ and $J=J_{1} \dot{\cup} J_{2}$, such that $|I|=3 m,\left|J_{1}\right|=\left|J_{2}\right|=2 m$, and for any $\bar{J} \subseteq J_{1}$, with $|\bar{J}|=m$, the induced graph $T[V(T) \backslash \bar{J}]$ has a perfect matching. We call $J_{1}$ the flexible set of vertices for the template.

Sparse templates, with maximum degree smaller than some absolute constant, are very useful in absorption arguments and can be used to design robust absorbing structures. Montgomery first introduced the use of such templates when applying the absorbing method in his work on spanning trees in random graphs [40]. Ferber, Kronenberg and Luh [21] followed the same argument as Montgomery (with some small adjustments) when studying the 2-universality of the random graph. Kwan [37] also used sparse templates to study random Steiner triple systems, generalising the template to a hypergraph setting and using it to define an absorbing structure for perfect matchings. Further applications were given by Ferber and Nenadov [22] in their work on universality in the random graph and recently by the current authors in [24] which was the first use of the method in the context of pseudorandom graphs, and by Nenadov and Pehova [42] who used the method to study a variant of the Hajnal-Szeméredi Theorem. The final three papers mentioned all adapt the method to give absorbing structures which output disjoint copies of a fixed graph $H$ (a partial $H$-factor), however the different absorbing structures used are interestingly all significantly distinct.

It is not difficult to prove the existence of sparse templates for large enough $m$ probabilistically; see e.g. [40, Lemma 2.8]. As we wish to give a completely algorithmic proof, in this section we show how to build a template $T$ efficiently. We use the following result of Lubotzky, Phillips and Sarnak [38].

Theorem 3.10. [38] For primes $p, q \equiv 1(\bmod 4)$ such that $p$ is a quadratic residue modulo $q$, one can construct an explicit $(p+1)$-regular Ramanujan graph $G$ in polynomial time (in $q)$ with $\left(q^{3}-q\right) / 2$ vertices. 
A Ramanujan graph, by definition, is a $d$-regular graph all of whose eigenvalues (other than $d$ and, if bipartite, $-d$ ) are in absolute value at most $2 \sqrt{d-1}$. We will in fact use a bipartite Ramanujan graph constructed as follows. Consider the graph $G$ provided by Theorem 3.10 - take $V_{1}$ and $V_{2}$ as two identical copies of $V(G)$, and join $v_{1} \in V_{1}$ and $v_{2} \in V_{2}$ if and only if the preimages of $v_{1}$ and $v_{2}$ in $V(G)$ form an edge of $G$. It is clear that this bipartite Ramanujan graph is still $d$-regular and satisfies the expander mixing lemma (1) for all $A \subseteq V_{1}$ and $B \subseteq V_{2}$, where $n$ is the number of vertices in each part, and $\lambda=2 \sqrt{d-1}$.

Proposition 3.11. Let $d \geq 144 / \alpha^{2}$. Let $G$ be a bipartite $d$-regular Ramanujan graph on vertex set $V_{1} \dot{\cup} V_{2}$, with $\left|V_{1}\right|=\left|V_{2}\right|=n$. Suppose $U \subseteq V_{1}$ and $W \subseteq V_{2}$ are vertex subsets of $V(G)$ such that $|U|=|W|=\alpha n$ and $\operatorname{deg}(w, U) \geq \alpha d / 3$ for any $w \in W$ and $\operatorname{deg}(u, W) \geq \alpha d / 3$ for any $u \in U$. Then $G[U, W]$ contains a perfect matching.

Proof. We will verify Hall's condition for $G[U, W]$. Note that it suffices to consider a set $X \subseteq U$ of size $|X| \leq|U| / 2=\alpha n / 2$. Let $Y=N(X) \cap W$ and we aim to show that $|Y| \geq|X|$. Assume to the contrary that $|Y|<|X|$. We first assume that $|X| \leq \alpha n / 6$. By the degree condition, we obtain that $e(X, Y) \geq|X| \alpha d / 3$. On the other hand, by (1), we have

$$
e(X, Y) \leq \frac{d}{n}|X||Y|+\lambda \sqrt{|X||Y|}<\frac{\alpha d}{6}|X|+2 \sqrt{d|X||Y|} .
$$

Putting these together, we get $2 \sqrt{d|X||Y|} \geq \alpha d|X| / 6$. By $d \geq 144 / \alpha^{2}$, this implies $|Y| \geq|X|$, a contradiction. Next we assume that $\alpha n / 6<|X| \leq \alpha n / 2$. By $|W \backslash Y| \geq \alpha n / 2$ and (1), we have

$$
e(X, W \backslash Y) \geq\left(\frac{d}{n} \sqrt{|X||W \backslash Y|}-\lambda\right)(\sqrt{|X||W \backslash Y|})>\alpha^{2} d n / 12-2 \sqrt{d \alpha^{2} n^{2} / 12},
$$

where the second inequality follows from the fact that both factors in the product are always positive, given the restraints on $|X|$ and $|W \backslash Y|$ and thus the product is minimised when the factors, and hence $|X|,|W \backslash Y|$, are as small as possible. Since $\alpha^{2} d \geq 144$, we obtain that $e(X, W \backslash Y)>0$, contradicting the definition of $Y$.

Lemma 3.12. Let $p \equiv 1(\bmod 4)$ be a prime such that $p \geq 68000$. For a sufficiently large integer $m$, we can construct a template with flexibility $m$ and maximum degree $d:=p+1$ in polynomial time.

Proof. It follows from the Siegel-Walfisz theorem [47] that we can pick a prime $q \equiv 1(\bmod 4 p)$ between $(21 m)^{1 / 3}$ and $1.01(21 m)^{1 / 3}$ for sufficiently large $m$. Thus $20 m \leq q^{3}-q \leq 22 m$. Using quadratic reciprocity to infer that $p$ is a quadratic residue modulo $q$, we have by Theorem 3.10 that we can construct in polynomial time a bipartite $d$-regular Ramanujan graph $G=(X \cup Y, E)$ with $10 m \leq|X|=|Y| \leq 11 \mathrm{~m}$ and second eigenvalue $\lambda \leq 2 \sqrt{d}$. We first show that for any set $U \subseteq X$ (or $Y$ ) of size at least $3 \mathrm{~m} / 2$, there are at most $34000 \mathrm{~m} / d$ vertices $v$ in $Y$ (or $X$ ) such that $\operatorname{deg}(v, U)<d / 10$. Indeed, denote by $B$ the set of such vertices $v$. Clearly we have $e(U, B)<d|B| / 10$. On the other hand, by (1), we have

$$
\frac{d|B|}{10}>e(U, B) \geq \frac{d}{11 m}|B||U|-\lambda \sqrt{|B||U|} \geq \frac{3 d|B|}{22}-2 \sqrt{d|B| \cdot 11 m} .
$$

This implies that $2 d|B| / 55<2 \sqrt{11 d|B| m}$, that is, $|B|<33275 m / d<34000 m / d$, as claimed. 
Now take arbitrary sets $V_{1}^{\prime \prime} \subseteq X, V_{2}^{\prime \prime} \subseteq Y$ such that $\left|V_{1}^{\prime \prime}\right|=3 m$ and $\left|V_{2}^{\prime \prime}\right|=2 m$. Next, we sequentially delete vertices from $V_{1}^{\prime \prime}$ and $V_{2}^{\prime \prime}$ as follows.

- Initiate with $V_{i}^{\prime}:=V_{i}^{\prime \prime}$ for $i=1,2$.

- If there is a vertex $v \in V_{1}^{\prime} \operatorname{such}$ that $\operatorname{deg}\left(v, V_{2}^{\prime}\right)<d / 10$, then delete $v$ from $V_{1}^{\prime}$,

- If there is a vertex $v \in V_{2}^{\prime} \operatorname{such}$ that $\operatorname{deg}\left(v, V_{1}^{\prime}\right)<d / 10$, then delete $v$ from $V_{2}^{\prime}$.

Note that since $\left|V_{i}^{\prime \prime}\right|-34000 \mathrm{~m} / d \geq 3 \mathrm{~m} / 2$, by our claim above, at most $34000 \mathrm{~m} / d$ vertices will be deleted from each set. Denote by $V_{1}^{\prime}$ and $V_{2}^{\prime}$ the resulting sets. Next, since there are at most $34000 \mathrm{~m} / d$ vertices that have degree less than $d / 10$ to $V_{i}^{\prime}, i=1,2$, respectively, we can add vertices to $V_{1}^{\prime}$ and $V_{2}^{\prime}$ and obtain $V_{1}$ and $V_{2}$ such that $\left|V_{1}\right|=3 m,\left|V_{2}\right|=2 m$ and $\operatorname{deg}\left(v, V_{i}\right) \geq d / 10$ for any $v \in V_{3-i}, i=1,2$. Finally, we pick $J_{1}$ as a set of $2 m$ vertices in $Y \backslash V_{2}$ which have degree at least $d / 10$ to $V_{1}$.

We claim that $T=G\left[V_{1} \cup V_{2} \cup J_{1}\right]$ is the desired template with flexible set $J_{1}$. It remains to check the property of $T$. For this, take any set $J^{\prime}$ of $m$ vertices in $J_{1}$ and consider $G\left[V_{1}, V_{2} \cup J^{\prime}\right]$. Since the assumptions of Proposition 3.11 are satisfied with $\alpha=3 m /|X| \epsilon[3 / 11,3 / 10], G\left[V_{1}, V_{2} \cup J^{\prime}\right]$ has a perfect matching and we are done. For the running time, note that in each of the steps above, it is enough to query the neighbourhood of a vertex, which can be done in constant time. So the overall running time is polynomial in $m$.

\section{Proof of Theorem 2.2}

In [24] an absorbing structure for cliques was defined. Here we generalise it for cycles as follows. Assume that $T=\left(I, J_{1} \cup J_{2}, E\right)$ is a bipartite template with flexibility $m$, maximum degree $\Delta(T) \leq K$ and flexible set $J_{1}$. It will be convenient to identify $T$ with its edges which may be viewed as the corresponding subset of tuples $(i, j) \in[3 m] \times[4 m]$, hence we will also think of $I$ as $[3 m], J_{1}$ as $[2 m], J_{2}$ as $[2 m+1,4 m]:=\{2 m+1, \ldots, 4 m\}$ and $J=J_{1} \cup J_{2}$.

An absorbing structure for cycles of length $s+2$ is a tuple $\mathcal{S}=\left(T, \mathcal{P}_{1}, A, \mathcal{P}_{2}, Z, Z_{1}\right)$ which consists of the template $T$ with flexibility $m$, the two sets $\mathcal{P}_{1}$ and $\mathcal{P}_{2}$ of vertex-disjoint paths of fixed length $s$ and three vertex sets $A, Z$ and $Z_{1}$ with $Z_{1} \subseteq Z$. Furthermore, the sets $V\left(\mathcal{P}_{1}\right), V\left(\mathcal{P}_{2}\right), A$ and $Z$ are pairwise disjoint and with the labelling $Z_{1}=\left\{z_{1}, \ldots, z_{2 m}\right\}, Z_{2}=\left\{z_{2 m+1}, \ldots, z_{4 m}\right\}$ (so that $\left.Z:=Z_{1} \cup Z_{2}\right), \mathcal{P}_{1}:=\left\{P^{1}, P^{2}, \ldots, P^{3 m}\right\}, A=\left\{a_{i j}:(i, j) \in E(T)\right\}$ and $\mathcal{P}_{2}=\left\{P_{i j}:(i, j) \in E(T)\right\}$, the following holds in $G$ for $(i, j) \in E(T)$ :

- $a_{i j}$ is adjacent to the ends of $P^{i}$, i.e. closes a cycle on $s+2$ vertices,

- each $a_{i j}$ is adjacent to the ends of $P_{i j}$,

- each $z_{j}$ is adjacent to the ends of $P_{i j}$.

In the proof of the following fact, we use a result of Micali and Vazirani [39], which constructs a maximum matching in general graphs in $O\left(|E||V|^{1 / 2}\right)$ time.

Fact 4.1. The absorbing structure $\mathcal{S}=\left(T, \mathcal{P}_{1}, A, \mathcal{P}_{2}, Z, Z_{1}\right)$ has the property that, for any subset $\bar{Z} \subseteq Z_{1}$ with $|\bar{Z}|=m$, the removal of $\bar{Z}$ leaves a graph with a $C_{s+2}$-factor, which can be found in time $O\left(m^{3 / 2}\right)$. 
Proof. By the property of the template $T \subseteq[3 m] \times[4 m]$, there is a perfect matching $M$ in $[3 m] \times$ $([4 m] \backslash \bar{J}) \cap T$ with $\bar{J}:=\left\{j: z_{j} \in \bar{Z}\right\}$. The above result from [39] finds $M$ in time $O\left(m^{3 / 2}\right)$.

Then for each edge $(i, j) \in M$, we take the $(s+2)$-cycles on $\left\{a_{i j}\right\} \cup P^{i}$ and $\left\{z_{j}\right\} \cup P_{i j}$; for the edges $(i, j) \in E(T) \backslash M$, we take the $(s+2)$-cycle on $\left\{a_{i j}\right\} \cup P_{i j}$. This gives the desired $C_{s+2}$-factor.

The following lemma is a variant of Lemma 2.7 from [24].

Lemma 4.2. Let $K:=68042$. For every $\delta>0, \ell \geq 4$ and $\alpha \in(0, \alpha(\ell)]($ where $\alpha(\ell):=1 /(60 \ell(K+2)))$ there exists $\varepsilon_{0}>0$ such that for all $\varepsilon \in\left(0, \varepsilon_{0}\right)$ there is an $n_{0} \in \mathbb{N}$ such that the following holds for all $n \geq n_{0}$. Let $G$ be a $(p, \lambda)$-graph with $n$ vertices, $p \in(0,1 / 3], \lambda \leq \varepsilon p^{2} n, \delta(G) \geq \delta p n$ and suppose $m=\alpha n$. Then in polynomial time we can find an absorbing structure $\mathcal{S}=\left(T, \mathcal{P}_{1}, A, \mathcal{P}_{2}, Z, Z_{1}\right)$ for cycles of length $\ell$ with flexibility $m$ in $G$. Further, one can find such an $\mathcal{S}$ such that there is a set $W \subseteq V(G) \backslash V(\mathcal{S})$, with $|W|=n / 4$ and $\operatorname{deg}(v, W) \geq \delta p|W| / 8$ for all vertices $v$ of $G$.

Proof. First we choose $\varepsilon_{0}=\min \left\{\delta /(400 K \ell), 2^{-(\ell+6)}, \alpha\right\}$ and let $\varepsilon \in\left(0, \varepsilon_{0}\right)$. Then we take $n_{0}$ large enough. Therefore, owing to Proposition 3.2, quantities $p^{2} n$ and $p n$ are large as well.

We consider a partition of $V(G)=V_{1} \dot{\cup} V_{2} \dot{\cup} V_{3} \dot{\cup} V_{4}$ with $\left|V_{1}\right|=\left|V_{2}\right|=\left|V_{3}\right|=\left|V_{4}\right|=n / 4$, such that

$$
\operatorname{deg}\left(v, V_{i}\right) \geq \delta p\left|V_{i}\right| / 2
$$

for all $i \in[4]$ and $v \in V$, as given by Corollary 3.8. We fix $W=V_{4}$ and thus the conditions on $W$ are satisfied. We now build our absorbing structure using vertices of $V(G) \backslash W$. Throughout the proof, we denote the intermediate partial absorbing structure by $\mathcal{S}^{\prime}$. Note that an absorbing structure for cycles of length $\ell$ with flexibility $m$ which uses a template $T$ has at most $3 \ell m(\Delta(T)+2)$ vertices, and thus, due to the condition on $\alpha$ and the fact that we will have $\Delta(T) \leq K$, we will have that $\left|V\left(\mathcal{S}^{\prime}\right)\right| \leq n / 20$ throughout the proof.

Let $T \subseteq[3 m] \times[4 m]$ be a bipartite template with flexibility $m$ and flexible set $J_{1}=[2 m]$ such that $\Delta(T) \leq K$, as provided by Lemma 3.12. Pick an arbitrary collection of $3 m$ vertex-disjoint copies of $C_{\ell}(1, \ldots, 1, K)$ in $V_{1}$ (using Fact 3.5$)$. For the $i$ th copy of $C_{\ell}(1, \ldots, 1, K)$, we label the corresponding path on $\ell-2$ edges by $P^{i}$ (so that the ends of $P^{i}$ have $K$ common neighbours), and we set $\mathcal{P}_{1}:=\left\{P^{1}, P^{2}, \ldots, P^{3 m}\right\}$. Then we label $A=\left\{a_{i j}:(i, j) \in E(T)\right\}$ as the vertices in the classes of $K$ vertices in the copies of $C_{\ell}(1, \ldots, 1, K)$ such that each $a_{i j}$ is connected to the ends of $P^{i}$, i.e. forms a copy of $C_{\ell}$ (we may then discard some extra vertices, according to the degree of $x_{i}$ in $T$ ).

We will pick $Z=\left\{z_{1}, \ldots, z_{4 m}\right\}$ and $\mathcal{P}_{2}=\left\{P_{i j}:(i, j) \in E(T)\right\}$ satisfying the definition of the absorbing structure as follows. We choose $Z$ in two phases, where all but at most $\varepsilon p^{2} n$ vertices for $Z$ will be chosen in the first phase. We first use vertices in $V_{1}$. We recursively do the following. We pick the smallest index $j \in[4 m]$ (as long as there exists such an index) so that $\left|N_{G}\left(a_{i j}, V_{1}\right) \backslash V\left(\mathcal{S}^{\prime}\right)\right| \geq$ $\delta p n / 10$ for all $i$ such that $(i, j) \in T$ (there are at most $K$ such $i$ ). We pick as $z_{j}$ an arbitrary vertex in $V_{2} \backslash\left(V\left(\mathcal{S}^{\prime}\right) \cup B_{j}\right)$, where $B_{j}$ is the set of vertices $z$ in $G$ such that $\left|\left(N_{G}\left(a_{i j}, V_{1}\right) \backslash V\left(\mathcal{S}^{\prime}\right)\right) \cap N_{G}(z)\right|<$ $\delta p^{2} n / 20$ for some $i$ with $(i, j) \in E(T)$. Since $\left|N_{G}\left(a_{i j}, V_{1}\right) \backslash V\left(\mathcal{S}^{\prime}\right)\right| \geq \delta p n / 10$ and $\Delta(T) \leq K$, Fact $3.3(i)$ with $U=N_{G}\left(a_{i j}, V_{1}\right) \backslash V\left(\mathcal{S}^{\prime}\right)$ implies that $\left|B_{j}\right| \leq 40 K \delta^{-1} \varepsilon^{2} p n \leq n / 8$, and so such a choice always exists.

Having chosen $z_{j}$, our next aim is to construct vertex-disjoint paths $P_{i j}$ of length $\ell-2$, for each $(i, j) \in E(T)$, so that the endpoints of $P_{i j}$ are adjacent to both $a_{i j}$ and $z_{j}$. For this purpose, we 
would like to pick two vertices $y_{1}, y_{2}$ in $U_{i j}:=\left(N_{G}\left(a_{i j}, V_{1}\right) \backslash V\left(\mathcal{S}^{\prime}\right)\right) \cap N_{G}\left(z_{j}\right)$, which are supposed to be the ends of the path $P_{i j}$ which we are going to construct. Since $z_{j} \notin B_{j}$, we have $\left|U_{i j}\right| \geq$ $\delta p^{2} n / 20$. Letting $V_{1}^{\prime}:=V_{1} \backslash\left(V\left(\mathcal{S}^{\prime}\right) \cup U_{i j}\right)$, we have that $\left|V_{1}^{\prime}\right| \geq n / 8$. From Remark 3.1, we get that $e\left(V_{1}^{\prime}, U_{i j}\right) \geq p\left|U_{i j}\right|\left|V_{1}^{\prime}\right| / 2$. We consider two cases. If $\ell=4$ then, since there is a vertex $w$ from $V_{1}^{\prime}$ of degree at least $p\left|U_{i j}\right| / 2 \geq \delta p^{3} n / 40 \geq 2$ into $U_{i j}$ (by Proposition 3.2), there is a path $P_{i j}$ of length 2 with ends (labeled as) $y_{1}$ and $y_{2}$ in $U_{i j}$. If $\ell \geq 5$, then by Fact $3.3(i)$ and the choice of $\varepsilon$, we can find two vertices $y_{1}$ and $y_{2} \in U_{i j}$, whose degrees into $V_{1}^{\prime}$ are at least $p n / 30$. Proposition 3.4 then yields the existence of a path of length $\ell-4$ with ends in $N\left(y_{1}\right) \cap V_{1}^{\prime}$ and $N\left(y_{2}\right) \cap V_{1}^{\prime}$. Together with $y_{1}$ and $y_{2}$ this provides us with the desired path $P_{i j}$.

It remains still to deal with the situation (second phase), when there are no remaining appropriate indices $j \in[4 m]$. Let $\tilde{J} \subseteq[4 m]$ be the set of those indices $j$ such that for some $\{i, j\} \in T$ we have $\left|N_{G}\left(a_{i j}, V_{1}\right) \backslash V\left(\mathcal{S}^{\prime}\right)\right|<\delta p n / 10$. Since $\left|V_{1} \backslash V\left(\mathcal{S}^{\prime}\right)\right| \geq n / 5$ we have with Fact $3.3(i)$ and $\Delta(T) \leq K$ that $|\tilde{J}| \leq K\left(20 \varepsilon^{2} p^{2} n\right) \leq \varepsilon p^{2} n$. To finish the embedding, we will use vertices in $V_{3}$ as well. At any point we will have that $\left|V\left(\mathcal{S}^{\prime}\right) \cap V_{3}\right| \leq K|\tilde{J}| \ell \leq \delta p n / 40$. From (3) we get $\operatorname{deg}\left(v, V_{3} \backslash V\left(\mathcal{S}^{\prime}\right)\right) \geq \delta p n / 10$ for all vertices $v \in V(G)$ throughout the process and we can proceed as in the two paragraphs above, using $V_{3}$ in place of $V_{1}$.

Now we analyse the running time. Firstly, we pick the copies of $C_{\ell}(1, \ldots, 1, K)$ by Fact 3.5 in time $O\left(p^{2} n^{3}\right)$. Secondly, to find a desired $j \in[4 m]$, we check $\left|N_{G}\left(a_{i j}, V_{1}\right) \backslash V\left(\mathcal{S}^{\prime}\right)\right|$ for all vertices $a_{i j}$, which takes time $O\left(p n^{2}\right)$; with such a $j$, to choose $z_{j}$, we search through the vertices $z$ not in $V\left(\mathcal{S}^{\prime}\right)$ and check $\left|\left(N_{G}\left(a_{i j}, V_{1}\right) \backslash V\left(\mathcal{S}^{\prime}\right)\right) \cap N_{G}(z)\right|$ for at most $K$ such $i$ 's. By Fact $3.3(i i)$, this takes time $O\left(p^{2} n^{2}\right)$. At last, we pick the desired path $P_{i j}$ of length $\ell-2$. If $\ell=4$, then we find the vertex $w \in V_{1}^{\prime}$ with degree 2 to $U_{i j}$, in time $O(n)$. So we find the path $P_{i j}$ in time $O\left(p n^{2}\right)$. If $\ell \geq 5$, we find $y_{1}$ and $y_{2}$ in time $\left|U_{i j}\right| \cdot O(p n)=O\left(p n^{2}\right)$ and apply Proposition 3.4, which runs in time $O\left(p^{2} n^{2}\right)$. The overall running time is polynomial since $O\left(p^{2} n^{3}\right)+O(m) \cdot O\left(p n^{2}\right)=O\left(p n^{3}\right)$ and partitioning as is done by Corollary 3.8 works in polynomial time as well.

Now we are ready to prove Theorem 2.2.

Proof of Theorem 2.2. Let $K=68042$. Let $L_{0}:=\min \left\{2^{k}: k \in \mathbb{N}, 2^{k}>L\right\}$ and fix $\varepsilon \leq \varepsilon_{0}:=$ $\min \left\{\delta /\left(8000 K L_{0}^{3} 2^{\left(L_{0}+6\right)}\right), \varepsilon_{4.2}, \varepsilon_{3.9}\right\}$, where $\varepsilon_{4.2}$ is as asserted by Lemma 4.2 on input $\delta^{\prime}:=\delta / 2 L_{0}$, $\alpha(L)$ and $\varepsilon_{3.9}$ is as asserted by Lemma 3.9 on input $\beta=1 /(60 L(K+2)), \delta^{\prime}$ and $L$. Let $n_{0}$ be large enough. First, using Corollary 3.8, we find a partition of the vertex set of $G$ into sets $V_{1} \cup V_{2}$ such that $\left|V_{1}\right|=n / L_{0}$ and every vertex $v \in V(G)$ satisfies

$$
\operatorname{deg}\left(v, V_{i}\right) \geq \delta p\left|V_{i}\right| / 2,
$$

for $i \in[2]$. Here $V_{2}$ is taken to be the union of all other sets in the equipartition given by Corollary 3.8 , thus $\left|V_{2}\right|=\left(L_{0}-1\right) n / L_{0}$. Let $F$ be a collection of cycles of lengths in the interval $[4, L]$, whose lengths sum up ${ }^{6}$ to $n$. There is (at least) one length $\ell \in[4, L]$ such that $F$ contains at least $n /((L-3) \ell)$ cycles $C_{\ell}$. We write $F=F^{\prime} \dot{\cup} F_{\ell}$, where $F_{\ell}$ consists of cycles of length $\ell$ from $F$, while $F^{\prime}$ contains all other cycles. We will embed $F$ into $G$ in two stages. First, we greedily embed $F^{\prime}$

${ }^{6}$ We can assume that $F$ has $n$ vertices as if not, we can take a supergraph by adding 4-cycles repeatedly. We can then remove up to three vertices from $G$ without affecting the properties of $G$ as in the statement of Theorem 2.2 . 
into $G\left[V_{2}\right]$. This is possible since

$$
\left|V\left(F^{\prime}\right)\right| \leq \frac{(L-4) n}{L-3} \leq \frac{\left(L_{0}-4\right) n}{L_{0}-3}=\frac{\left(L_{0}-1\right) n}{L_{0}}-\frac{3 n}{L_{0}\left(L_{0}-3\right)}=\left|V_{2}\right|-\frac{3 n}{L_{0}\left(L_{0}-3\right)}
$$

and since any set of at least $3 n /\left(L_{0}\left(L_{0}-3\right)\right)$ vertices in $G$ contains a cycle of any length from the interval $[4, L]$ (see Fact 3.5).

In the second stage we are left with a vertex set $U \supseteq V_{1}$ such that $\left|V\left(F_{\ell}\right)\right|=|U|$ and $\delta(G[U]) \geq$ $\delta p n /\left(2 L_{0}\right) \geq \delta^{\prime} p|U|$, due to (4). All that remains to do is to find a $C_{\ell}$-factor in $G[U]$. We are thus in a position to apply Lemma 4.2 to $G[U]$, where one can check that the conditions there are satisfied with respect to $|U|$ and $\delta^{\prime}$. Thus, in polynomial time we can construct an absorbing structure $\mathcal{S}=\left(T, \mathcal{P}_{1}, A, \mathcal{P}_{2}, Z, Z_{1}\right)$ for cycles of length $\ell$ with flexibility $m=\alpha|U|$, where $\alpha:=1 /(60 L(K+2)) \leq$ $\alpha(\ell)$, and a vertex set $W \subseteq V(G) \backslash V(\mathcal{S})$, with $|W|=|U| / 4$, such that for any vertex $v$ in $G$, we have $\operatorname{deg}(v, W) \geq \delta^{\prime} p|U| / 8$. Let $U_{0} \subseteq(U \backslash V(\mathcal{S}))$ be the set of vertices $u$ such that $\operatorname{deg}\left(u, Z_{1}\right) \leq p\left|Z_{1}\right| / 2$. By Fact $3.3(i)$, we have that $\left|U_{0}\right| \leq 4 \varepsilon^{2} p^{2}|U|^{2} /\left|Z_{1}\right|=2 \varepsilon^{2} \alpha^{-1} p^{2}|U|$. We first incorporate the vertices of $U_{0}$ into cycles of length $\ell$ using vertices of $W \backslash U_{0}$ by applying Lemma 3.9 (in polynomial time) to the pairs $\left\{(u, u): u \in U_{0}\right\}$. Let $\mathcal{C}_{1}$ be the set of disjoint cycles produced by this process.

Now we greedily apply Fact 3.5 to find vertex-disjoint cycles $C_{\ell}$ in $G\left[U \backslash\left(V(\mathcal{S}) \cup V\left(\mathcal{C}_{1}\right)\right)\right]$, until we are left with a set $U_{1}$ of cardinality at most $2^{\ell} \varepsilon n$. What remains is to find a $C_{\ell}$-factor in $G\left[U_{1} \dot{\cup} V(\mathcal{S})\right]$. Recall that $\operatorname{deg}\left(u, Z_{1}\right) \geq p\left|Z_{1}\right| / 2$ for every $u \in U_{1}$. The assumptions of Lemma 3.9 are met (in particular $\left|Z_{1}\right| \gg\left|U_{1}\right|$ ), and therefore, applying it to the pairs of vertices $\left\{(u, u): u \in U_{1}\right\}$ (to find paths through $Z_{1}$ ) we find a family $\mathcal{C}_{2}$ of $\left|U_{1}\right|$ vertex-disjoint cycles $C_{\ell}$ that cover all of $U_{1}$ (and some subset of $Z_{1}$ ). Next, we greedily find, applying Fact 3.5, $\left(m-\left|U_{1}\right|(\ell-1)\right) / \ell$ cycles $C_{\ell}$ in $Z_{1} \backslash V\left(\mathcal{C}_{2}\right)$, so a set $Z_{1}^{\prime}$ of exactly $m$ vertices of $Z_{1}$ remains uncovered. But then, letting $Z_{1}^{\prime \prime}=Z_{1} \backslash Z_{1}^{\prime}$, Fact 4.1 guarantees the existence of a $C_{\ell}$-factor on $V(\mathcal{S}) \backslash Z_{1}^{\prime \prime}$. This then gives us a copy of $F$ in $G$.

Note that we applied Fact 3.5 linearly many times, which took $O\left(p^{2} n^{3}\right)$ running time. Moreover, we applied Corollary 3.8, Lemma 3.9, Fact 4.1 and Lemma 4.2 constantly many times. So we conclude that we can indeed find a copy of $F$ in polynomial time.

\section{Proof of Theorem 2.3}

Before proving Theorem 2.3, let us sketch some of the ideas that arise in the proof. Firstly we will apply Lemma 4.2 to show the existence of an absorbing structure $\mathcal{S}=\left(T, \mathcal{P}_{1}, A, \mathcal{P}_{2}, Z, Z_{1}\right)$ for cycles of length 4 with flexibility $m=\lfloor\gamma n\rfloor$, with $\gamma \leq \alpha(4)=1 /(240(K+2))$, as defined in Lemma 4.2. Recall that Fact 4.1 guarantees that no matter which $m$ vertices of $Z_{1}$ we remove, on the rest of the vertices of $\mathcal{S}$ we can find a $C_{4}$-factor (which will contain exactly $3 m+|E(T)|$ copies of $\left.C_{4}\right)$. Let us relabel the $r:=3 m+|E(T)|$ paths of length two in $\mathcal{P}_{1} \cup \mathcal{P}_{2}$ as $\mathcal{Q}=\left\{Q_{1}, Q_{2}, \ldots, Q_{r}\right\}$, let $Q_{h}=a_{h} b_{h} c_{h}$ for each $h \in[r]$ and let $Y=Z \dot{\cup} A$. Now the property of the absorbing structure can be rephrased as follows. After removing exactly $m$ vertices, $Z^{\prime}$, from $Z_{1} \subseteq Y$, there is a perfect matching between $\mathcal{Q}$ and $Y \backslash Z^{\prime}$ such that if $Q_{h} \in \mathcal{Q}$ is matched with $y \in Y$, then $a_{h} y c_{h} b_{h}$ forms a copy of $C_{4}$. In what follows, the idea is to omit an edge (for example, $a_{h} b_{h}$ ) from each of these $C_{4}$ to get paths of length three which we will connect to longer paths. The key point is that we can do 
this by only omitting edges in the length two paths from $\mathcal{Q}$. Thus we can simply connect vertices from paths in $\mathcal{Q}$ through short connecting paths. Eventually, this will lead to a longer path that will contribute to our factor and although we do not know exactly what these paths will be (as it depends on the choice of matching to $y \in Y$ ), the lengths of the paths and the vertices not in $Y$ are fixed. More precisely, we will group the paths in $\mathcal{Q}$ according to the desired lengths of the cycle and connect the ones in the same group e.g. connect $a_{h}$ with $b_{h-1}$ and connect $b_{h}$ with $a_{h+1}$. At the end of the proof, by Fact 4.1 we can match every remaining vertex $y \in Y$ to one of the $Q_{h}$ 's, such that $a_{h} y c_{h} b_{h}$ forms a copy of $P_{3}$ which will contribute to some longer path which in turn is part of a cycle in $F$.

Our proof of Theorem 2.3 is algorithmic and we split the algorithm into three phases. Let us concentrate here on the case where $F$ is a full 2-factor i.e. $F$ has $n$ vertices. The first phase will build an initial segment for some of the cycles in our $F$, by finding short path segments which use the vertices of $\mathcal{Q}$ and also, with foresight, incorporate some vertices that may be troublesome in the last phase of our algorithm. Our second phase will incorporate the majority of the vertices into paths. For each cycle in $F$ we will greedily choose a path avoiding a fixed subset $Z^{\prime} \subseteq Z_{1}$ as well as $Z_{2}$ and the previously chosen vertices from the first phase. We will terminate this greedy phase with just $\varepsilon n$ candidate vertices not added to the paths. We will then use small paths through $Z^{\prime}$ to connect the initial path segments from phase one, the greedy paths from phase two and the $\varepsilon n$ remaining vertices. We will do this in such a way that we are left with $m$ vertices in $Z_{1}$, say $Z^{\prime \prime}$, and thus by the key property of our template $T$, there is a matching on $T\left[I \cup J^{\prime \prime}\right]$, where $J^{\prime \prime}=\left\{j \in[4 m]: z_{j} \in Z^{\prime \prime}\right\}$. This will dictate a matching between $Z^{\prime \prime} \cup A$ and $\mathcal{Q}$, which in turn tells us how to incorporate the vertices of $Z^{\prime \prime} \cup A$ into our cycles. This then results in disjoint cycles of the right size with every vertex used, that is, a copy of $F$ in $G$.

Proof of Theorem 2.3. Let $K=68042$. Let $L \geq 8000 K$ and fix $\gamma:=1 /(600(K+2)) \leq \alpha(4)$ with $\alpha(4)$ defined in Lemma 4.2. Next, choose $\varepsilon \leq \varepsilon_{1}:=\min \left\{\delta /(1600000 K), \varepsilon_{4.2}, \varepsilon_{3.9}\right\}$, where $\varepsilon_{4.2}$ is as asserted by Lemma 4.2 on input $\delta, \alpha=\gamma, \ell=4$ and $\varepsilon_{3.9}$ is as asserted by Lemma 3.9 on input $\beta=\gamma$, $\delta^{\prime}:=\delta / 16$ and $\ell=3$. Let $n_{0}$ be large enough. Let $F$ be a graph on $n$ vertices, whose components are cycles of length greater than $L$. We can assume that $v(F) \geq n-L$, otherwise we can instead consider a supergraph by adding cycles of length $L+1$. Let $F$ consist of $t$ cycles of lengths $l_{1} \geq \cdots \geq l_{t}$, and let $l=\sum_{i}^{t} l_{i}$. Note that $t \leq n / L$ and $n-L \leq l \leq n$. We will show that $F \subseteq G$.

Let $m=\gamma n$. Apply Lemma 4.2 to get an absorbing structure $\mathcal{S}=\left(T, \mathcal{P}_{1}, A, \mathcal{P}_{2}, Z, Z_{1}\right)$ for cycles of length 4 with flexibility $m$ and a vertex set $W \subseteq V(G) \backslash V(\mathcal{S})$, with $|W|=n / 4$, such that for any vertex $v$ in $G$, we have $\operatorname{deg}(v, W) \geq \delta p|W| / 8$. Label the vertices and paths of $\mathcal{S}$ as in the discussion above. In particular, recall that $r:=3 m+|E(T)|$. Let $m^{\prime}:=\varepsilon n$, and let $Z^{\prime} \subseteq Z_{1}$ be an arbitrary subset of size $m+2 m^{\prime}+4 t$. Let $V_{0} \subseteq(V(G) \backslash V(\mathcal{S})) \cup\left(Z_{1} \backslash Z^{\prime}\right)$ be the set of vertices $v$ such that $\operatorname{deg}\left(v, Z^{\prime}\right) \leq \frac{p\left|Z^{\prime}\right|}{2}$. Write $V_{0}:=\left\{v_{1}, v_{2}, \ldots, v_{\left|V_{0}\right|}\right\}$. By Fact $3.3(i)$, we have that $\left|V_{0}\right| \leq 4 \varepsilon^{2} \gamma^{-1} p^{2} n$. We find nonnegative integers $q_{i j}, i \in[t], j \in[3]$ such that the following holds:

- $6 q_{i 1}+3 q_{i 2}+3 q_{i 3} \leq l_{i}-10$, for each $i \in[t]$,

- $\sum_{i=1}^{t} q_{i 1}=r, \sum_{i=1}^{t} q_{i 2}=\left|V_{0}\right|$, and $\sum_{i=1}^{t} q_{i 3}=m^{\prime}$.

Such choice can be achieved easily since $r=3 m+|E(T)|$ and $6 r+3\left|V_{0}\right|+3 m^{\prime} \ll l-15 t$. 
We now run the first phase of our algorithm:

(i) We arbitrarily partition the set $\left\{\left\{a_{h}, b_{h}, c_{h}\right\}, h \in[r]\right\}$ into $t$ subsets of sizes $q_{11}, q_{21}, \ldots, q_{t 1}$ and partition $V_{0}$ into $t$ subsets of sizes $q_{12}, \ldots, q_{t 2}$.

(ii) For $i \in[t]$, we fix an arbitrary linear order of the $q_{i 1}$ triples of vertices and $q_{i 2}$ vertices of $V_{0}$, and insert two new vertices $x_{i}^{1}, x_{i}^{2}$ not in $W \cup V_{0} \cup V(\mathcal{S})$ to the ordering, one to the beginning, one to the end. Apply Lemma 3.9 to the pairs $\left\{b_{h-1}, a_{h}\right\}$ of consecutive elements from each group simultaneously (we view each single vertex $v$ in the ordering as $v=a_{h}=b_{h}$ ), and get disjoint length three paths through $W \backslash V_{0}$ joining the pairs. This is possible because the number of pairs we connect is at most $2 t+r+\left|V_{0}\right| \leq 2 n / L+3 m(1+K)+4 \varepsilon^{2} \gamma^{-1} p^{2} n \leq$ $2 n / L+3(K+2) \gamma n<n / 120$, and every vertex has degree at least $\delta p|W| / 8-\left|V_{0}\right| \geq \delta p|W| / 9$ to $W \backslash V_{0}$, and $\left|W \backslash V_{0}\right| \geq n / 5$.

For each $i \in[t]$, we obtain a sequence of paths on (in total) $5 q_{i 1}+3 q_{i 2}+3$ vertices (they will become a single path of length $6 q_{i 1}+3 q_{i 2}+3$ after absorbing exactly $q_{i 1}$ vertices from $Z_{1}$ ). Next we will greedily find paths for each $i \in[t]$ which will comprise the majority of the remainder of the cycles.

(iii) Fix $U$ to be the vertices in $(V(G) \backslash(V(\mathcal{S}))) \cup\left(Z_{1} \backslash Z^{\prime}\right)$ which were not used in the paths chosen in the first phase. For $i \in[t]$, we repeatedly find a path of length exactly $l_{i}-6 q_{i 1}-3 q_{i 2}-3 q_{i 3}-9$ in the uncovered vertices of $U$ using Lemma 3.6 (for this observe that there are at least $\geq \varepsilon n$ unused vertices from $U$ by the choice of the parameters). Denote the endpoints of the path by $x_{i}^{3}$ and $x_{i}^{4}$.

(iv) Arbitrarily choose $m^{\prime}$ vertices from $U$ (it could happen that there are more vertices in $U$ but only if $F$ has less than $n$ vertices), partition and label them in such a way that for each $i$ there are $q_{i 3}$ vertices $u_{i, 1}, \ldots, u_{i, q_{i 3}}$.

$(v)$ Apply Lemma 3.9 to find paths of length 3 to connect the following set of pairs

$$
\bigcup_{i=1}^{t}\left\{\left(x_{i}^{2}, x_{i}^{3}\right),\left(x_{i}^{4}, u_{i, 1}\right),\left(u_{i, 1}, u_{i, 2}\right), \ldots,\left(u_{i, q_{i 3}}, x_{i}^{1}\right)\right\}
$$

with inner vertices from $Z^{\prime}$. Note that this is possible as all the vertices of the pairs have good degree to $Z^{\prime}$ and the number of pairs to connect is $2 t+\sum_{i} q_{i 3}=2 t+m^{\prime}$, which is much less than $m=\gamma n$.

(vi) In the previous step we used exactly $2 m^{\prime}+4 t$ vertices of $Z^{\prime}$ in length 3 paths. Thus the set $Z^{\prime \prime} \subseteq Z_{1}$ of unused vertices has size exactly $m$. By Fact 4.1 we can find a $C_{4}$-factor on $V(\mathcal{S}) \backslash\left(Z_{1} \backslash Z^{\prime \prime}\right)$ in time $O\left(n^{3 / 2}\right)$. Note that the paths $a_{j} y_{j} c_{j} b_{j}$ for each $C_{4}$ on $\left\{y_{j}, a_{j}, b_{j}, c_{j}\right\}$ will complete the cycles of length exactly

$$
\left(6 q_{i 1}+3 q_{i 2}+3\right)+\left(l_{i}-6 q_{i 1}-3 q_{i 2}-3 q_{i 3}-9\right)+3 q_{i 3}+6=l_{i}
$$

for each $i \in[t]$. Thus, we have found a copy of $F$ in $G$.

Note that we can compute the values of $q_{i j}$ greedily in time $O(n)$. Each of Lemma 3.9, Fact 4.1 and Lemma 4.2 runs in polynomial time and we use them at most twice. Finally, we applied 
Lemma $3.6 t$ times. However, since the sum of the lengths of the paths we constructed is at most $n$, the running time is $O\left(p^{2} n^{3}\right)$. So the overall running time is polynomial.

Let us mention here that one could also define an absorbing structure specifically for the longer cycles we build in Theorem 2.3, connecting edges into paths according to the adjacencies of a template. Although this alternative structure would be easier to describe and would remove some of the technicalities in the above proof, we chose to instead work from the absorbing structure used for tiling with short cycles, for the sake of brevity.

\section{A proof of Theorem 2.1}

Nenadov's proof is algorithmic, except the proof of [41, Lemma 3.5], in which he used a Halltype result for hypergraphs due to Haxell. Here we give an alternative proof of this lemma, which moreover provides a polynomial time algorithm.

We first need to recall some definitions from [41]. Let $K_{4}^{-}$be the unique graph with 4 vertices and 5 edges. Define an $\ell$-chain as a graph obtained by sequentially identifying $\ell$ copies of $K_{4}^{-}$on vertices of degree 2 . Note that an $\ell$-chain contains exactly $\ell+1$ vertices such that the removal of any one of them results in a graph that has a triangle-factor. These vertices are called removable.

We say that a triangle in $G$ traverses three chains $D_{1}, D_{2}$ and $D_{3}$ if it intersects all of them at some removable vertices. Observe that if $D_{1}, D_{2}$ and $D_{3}$ are disjoint chains in $G$ and there exists a triangle in $G$ traversing them, then $G\left[V\left(D_{1}\right) \cup V\left(D_{2}\right) \cup V\left(D_{3}\right)\right]$ contains a triangle-factor.

Here we state [41, Lemma 3.5] and give an alternative (algorithmic) proof.

Lemma 6.1 (Lemma 3.5 in [41]). Let $G$ be a $(p, \lambda)$-bijumbled graph on $n$ vertices with $\lambda \leq \varepsilon p^{2} n$ for some $\varepsilon \in(0,1 / 16]$. Suppose we are given disjoint $\ell$-chains $D_{1}^{\prime}, \ldots, D_{t}^{\prime} \subseteq G$ for some $t, \ell \in \mathbb{N}$ such that $\ell$ is even, $t \geq 2000$ and $400 \lambda / p^{2} \leq t(\ell+1) \leq n / 24$. Then for any subset $W \subseteq V(G) \backslash \bigcup_{i \in[t]} V\left(D_{i}^{\prime}\right)$ of size $|W| \geq n / 4$ there exist disjoint $(\ell / 2)$-chains $D_{1}, \ldots, D_{2 t} \subseteq G[W]$ with the following property: for every $L \subseteq[2 t]$ there exists $L^{\prime} \subseteq[t]$ such that

$$
G\left[\bigcup_{i \in L} V\left(D_{i}\right) \cup \bigcup_{i \in L^{\prime}} V\left(D_{i}^{\prime}\right)\right]
$$

contains a triangle-factor, which can be found in polynomial time.

Proof. We set $\varepsilon:=1 / 16$. Note that a similar calculation as in the proof of Fact $3.3(i)$ shows that the number of vertices which have at most $\varepsilon p t \ell$ neighbours in a set of size at least $t(\ell+1) / 8 \geq 50 \lambda / p^{2}$ is at most

$$
\frac{\lambda^{2} t(\ell+1) / 8}{(1 / 8-\varepsilon)^{2} p^{2} t^{2}(\ell+1)^{2}} \leq \frac{\lambda}{3200(1 / 8-\varepsilon)^{2}}<\lambda / 2 .
$$

Given $\ell$-chains $D_{1}^{\prime}, \ldots, D_{t}^{\prime}$, we partition them arbitrarily into four groups of almost equal sizes, $\mathcal{D}_{1}, \ldots, \mathcal{D}_{4}$. Note that for $\mathcal{D}_{3}$ and $\mathcal{D}_{4}$, since each of them contains at least $t(\ell+1) / 4$ removable vertices, by (5) the number of vertices of $G$ that have degree less than $\varepsilon p t \ell \leq \varepsilon p n / 24$ to either of their removable vertices is at most $\lambda$. Now we greedily pick $2 t(\ell / 2)$-chains $D_{1}, \ldots, D_{2 t}$ in $W$ but avoiding these bad vertices by [41, Lemma 3.2]. It remains to verify the 'absorption' property. Fix any subset $L \subseteq[2 t]$ of $(\ell / 2)$-chains $D_{i}, i \in L$. We first greedily find triangles traversing $(\ell / 2)$-chains 
(and thus obtain triangle-factors on them) until $t / 8$ of them are left. Indeed, this is possible since as long as there are more than $t / 8$ of them left, we can greedily partition them into three groups of size roughly $t / 24$. Because $(t / 24)(\ell / 2+1)>t(\ell+1) / 48>2 \lambda / p^{2}$, it follows from (2) (for a proof see for example [41, Lemma 2.4]), we find a triangle with one vertex from each group. This triangle traverses the three chains containing it and thus there is a triangle-factor covering these three chains. So we can reduce the number of chains by 3 .

We will match the remaining $t / 8(\ell / 2)$-chains with the $\ell$-chains. We start with using $\ell$-chains in $\mathcal{D}_{1}, \mathcal{D}_{2}$ and recursively find triangles traversing one $(\ell / 2)$-chain and one $\ell$-chain in $\mathcal{D}_{1}$, one $\ell$-chain in $\mathcal{D}_{2}$. That is, as long as there exists a vertex $v$ in one of the 'unmatched' chains that sends more than $\varepsilon p t \ell$ edges to the unused removable vertices in both $\mathcal{D}_{1}$ and $\mathcal{D}_{2}$, then we pick an edge (whose existence is asserted by (2)) from these neighbourhoods, namely, a triangle containing $v$. Note that when we stop, the vertices remaining unmatched have degree at most $\varepsilon p t \ell$ to the unused removable vertices of either in $\mathcal{D}_{1}$ or $\mathcal{D}_{2}$. Note that there are still roughly half of the chains in $\mathcal{D}_{1}$ and $\mathcal{D}_{2}$ left, which contain at least $(\ell+1) \cdot t / 8$ removable vertices in both $\mathcal{D}_{1}$ and $\mathcal{D}_{2}$. Thus, by (5) there are at most $\lambda$ vertices that send low degree to either of them, namely, at most $2 \lambda / \ell(\ell / 2)$-chains are left unmatched. Now we can proceed to match the chains greedily by $\mathcal{D}_{3}$ and $\mathcal{D}_{4}$. This is possible because each time we match a chain, we consume $\ell+1$ removable vertices from $\mathcal{D}_{3}$ and $\mathcal{D}_{4}$, respectively, and so in total this will consume at most $(\ell+1)(2 \lambda / \ell)=2 \lambda(1+1 / \ell)$ removable vertices, which is much less than $\varepsilon p t \ell$.

For the running time, note that we used [41, Lemma 3.2] in the proof, but the desired chains can be constructed by depth-first search, which can be done in polynomial time. We also used [41, Lemma 2.4] to claim the existence of a triangle, but we can then find this triangle by brute-force searching the neighbourhood of a vertex, in time $O\left(p^{2} n^{3}\right)$. Finally, it takes time $O\left(n^{3}\right)$ to decide which $v$ to use and $O\left(p^{2} n^{2}\right)$ to find the triangle containing $v$. Thus, the greedy process can be done in time $O\left(n^{4}\right)$.

\section{Concluding Remarks}

In this paper we answered the question of Nenadov [41] by providing a deterministic polynomial time algorithm, which finds any given 2 -factor in a $\left(p, \varepsilon p^{2} n / \log n\right)$-bijumbled graph on $n$ vertices of minimum degree $\delta p n$ (for any fixed $\delta>0$ ), with $p>0$ and some absolute parameter $\varepsilon=\varepsilon(\delta)>$ 0 . This is optimal up to the $O(\log n)$-factor. It also follows from the proof that the strongest condition hinges on the fact that a triangle might be present in a 2-factor (see Theorem 2.1). Indeed, it follows from the proof of Theorem 2.2 that for a 2-factor of girth at least 4 , a weaker condition suffices. The celebrated construction, due to Alon [9], of triangle-free pseudorandom graphs has been extended by Alon and Kahale [12] to graphs without odd cycles of length $2 \ell+1$. They constructed $\left(n, \Theta\left(n^{2 /(2 \ell+1)}\right), \Theta\left(n^{1 /(2 \ell+1)}\right)\right)$-graphs of odd girth at least $2 \ell+3$. It is proved in [34, Proposition 4.12] that an $(n, d, \lambda)$-graph with $\lambda^{2 \ell-1} \ll d^{2 \ell} / n$ contains a copy of $C_{2 \ell+1}$. Since

$\lambda=\Omega(\sqrt{d})$ for, say $d \leq n / 2$, we have the lower bound on $d=\Omega\left(n^{2 /(2 \ell+1)}\right)$. As for even cycles, a theorem of Bondy and Simonovits [14], which doesn't require any bound on $\lambda$, states that $d \gg n^{1 / \ell}$ already implies the existence of $C_{2 \ell}$. It is thus a natural avenue to further investigate the (almost) 
optimal conditions of when a $(p, \lambda)$-bijumbled graph contains a given 2-factor of girth at least $\ell$. When $\ell=n$, the best condition for $(n, d, \lambda)$-graphs is provided by the result of Krivelevich and Sudakov [33] which gives $\lambda \leq d(\log \log n)^{2} /(1000 \log n \log \log \log n)$, while another conjecture of these authors [33] states that $\lambda \leq c d$ should already be sufficient for some absolute $c>0$. This conjecture would follow from the famous toughness conjecture of Chvátal [16], as shown by Alon [10].

\section{REFERENCES}

[1] P. Allen, J. Böttcher, H. Hàn, Y. Kohayakawa, and Y. Person, Blow-up lemmas for sparse graphs (2016), available at arXiv:1612.00622. $\uparrow 2$

[2] P. Allen, J. Böttcher, Y. Kohayakawa, and Y. Person, Tight Hamilton cycles in random hypergraphs, Random Struct. Algorithms 46 (2015), no. 3, 446-465. $\uparrow 4$

[3] P. Allen, J. Böttcher, H. Hàn, Y. Kohayakawa, and Y. Person, Powers of Hamilton cycles in pseudorandom graphs, Combinatorica 37 (2017), no. 4, 573-616. MR3694704 ^2, 3, 4

[4] N. Alon, Universality, tolerance, chaos and order, An irregular mind. Szemerédi is 70. Dedicated to Endre Szemerédi on the occasion of his seventieth birthday., 2010, pp. 21-37. $\uparrow 2$

[5] N. Alon and M. Capalbo, Sparse universal graphs for bounded-degree graphs, Random Struct. Algorithms 31 (2007), no. 2, 123-133. $\uparrow 2$

[6] _ Optimal universal graphs with deterministic embedding., Proceedings of the nineteenth annual ACMSIAM symposium on discrete algorithms (SODA 2008), San Francisco, CA, January 20-22, 2008, 2008, pp. 373378. $\uparrow 2$

[7] N. Alon, M. Capalbo, Y. Kohayakawa, V. Rödl, A. Ruciński, and E. Szemerédi, Universality and tolerance, Proceedings of the 41st IEEE FOCS, 2000, pp. 14-21. $\uparrow 2$

[8] N. Alon and F. R. K. Chung, Explicit construction of linear sized tolerant networks, Discrete Mathematics 72 (1988), no. 1-3, 15-19. MR90f:05080 ^1

[9] N. Alon, Explicit Ramsey graphs and orthonormal labelings, Electron. J. Combin. 1 (1994), Research Paper 12, 8 pp (electronic). MR1302331 (95k:05145) $\uparrow 3,18$

[10] _ Tough ramsey graphs without short cycles, Journal of Algebraic Combinatorics 4 (1995), no. 3, $189-195$. $\uparrow 19$

[11] N. Alon, M. Capalbo, Y. Kohayakawa, V. Rödl, A. Ruciński, and E. Szemerédi, Near-optimum universal graphs for graphs with bounded degrees (extended abstract), Approximation, randomization, and combinatorial optimization (Berkeley, CA, 2001), 2001, pp. 170-180. $\uparrow 4$

[12] N. Alon and N. Kahale, Approximating the independence number via the $\vartheta$-function, Mathematical Programming 80 (1998), no. 3, 253-264. $\uparrow 18$

[13] N. Alon and J. H. Spencer, The probabilistic method, Fourth, Wiley Series in Discrete Mathematics and Optimization, John Wiley \& Sons, Inc., Hoboken, NJ, 2016. MR3524748 ^8

[14] J. A. Bondy and M. Simonovits, Cycles of even length in graphs., J. Comb. Theory, Ser. B 16 (1974), 97-105. $\uparrow 18$

[15] F. R. K. Chung, R. L. Graham, and R. M. Wilson, Quasi-random graphs, Combinatorica 9 (1989), no. 4, 345-362. $\uparrow 2$

[16] V. Chvátal, Tough graphs and Hamiltonian circuits, Discrete Mathematics 5 (1973), no. 3, 215-228. $\uparrow 19$

[17] D. Conlon, A sequence of triangle-free pseudorandom graphs, Combinatorics, Probability and Computing 26 (2017), no. 2, 195-200. $\uparrow 3$

[18] D. Conlon, A. Ferber, R. Nenadov, and N. Škorić, Almost-spanning universality in random graphs, Random Struct. Algorithms 50 (2017), no. 3, 380-393. $\uparrow 2$

[19] D. Conlon, J. Fox, and Y. Zhao, Extremal results in sparse pseudorandom graphs, Adv. Math. 256 (2014), 206290. MR3177293 $\uparrow 2$ 
[20] D. Dellamonica Jr., Y. Kohayakawa, V. Rödl, and A. Ruciński, An improved upper bound on the density of universal random graphs, Latin 2012: Theoretical informatics, 2012, pp. 231-242. $\uparrow 2$

[21] A. Ferber, G. Kronenberg, and K. Luh, Optimal threshold for a random graph to be 2-universal, 2016. arXiv:1612.06026. $\uparrow 2,9$

[22] A. Ferber and R. Nenadov, Spanning universality in random graphs (2017), available at arXiv:1707.07914. $\uparrow 2,9$

[23] J. Han, Y. Kohayakawa, and Y. Person, Near-optimal clique-factors in sparse pseudorandom graphs. arXiv:1806.00493, submitted. $\uparrow 2,3$

[24] J. Han, Y. Kohayakawa, P. Morris, and Y. Person, Clique-factors in sparse pseudorandom graphs. arXiv:1806.01676, submitted. $\uparrow 2,4,9,11,12$

[25] J. Han, Y. Kohayakawa, and Y. Person, Near-perfect clique-factors in sparse pseudorandom graphs., Discrete mathematics days 2018. Extended abstracts of the 11th "Jornadas de matemática discreta y algorítmica" (JMDA), Sevilla, Spain, June 27-29, 2018, 2018, pp. 221-226. $\uparrow 3$

[26] S. Hoory, N. Linial, and A. Widgerson, Expander graphs and their applications., Bull. Am. Math. Soc., New Ser. 43 (2006), no. 4, 439-561. $\uparrow 2,4$

[27] J. H. Kim and S. J. Lee, Universality of random graphs for graphs of maximum degree two, SIAM Journal on Discrete Mathematics 28 (2014), no. 3, 1467-1478. $\uparrow 2$

[28] Y. Kohayakawa, V. Rödl, M. Schacht, P. Sissokho, and J. Skokan, Turán's theorem for pseudo-random graphs, J. Combin. Theory Ser. A 114 (2007), no. 4, 631-657. MR2319167 ^3

[29] J. Komlós, G. N. Sárközy, and E. Szemerédi, Blow-up lemma, Combinatorica 17 (1997), no. 1, $109-123$. MR99b:05083 $\uparrow 2$

[30] J. Komlós, G. N. Sárközy, and E. Szemerédi, An algorithmic version of the blow-up lemma., Random Struct. Algorithms 12 (1998), no. 3, 297-312. $\uparrow 5$

[31] J. Komlós, A. Shokoufandeh, M. Simonovits, and E. Szemerédi, The regularity lemma and its applications in graph theory., Theoretical aspects of computer science. Advanced lectures, 2002, pp. 84-112. $\uparrow 2$

[32] S. Kopparty, Cayley graphs (lecture notes), Rutgers University, $2011 . \quad$ available: http://sites.math.rutgers.edu/ sk1233/courses/graphtheory-F11/cayley.pdf. $\uparrow 3$

[33] M. Krivelevich and B. Sudakov, Sparse pseudo-random graphs are Hamiltonian, J. Graph Theory 42 (2003), no. 1, 17-33. MR1943104 (2003j:05114) $\uparrow 2,19$

[34] _ Pseudo-random graphs, More sets, graphs and numbers, 2006, pp. 199-262. MR2223394 (2007a:05130) $\uparrow 2,18$

[35] M. Krivelevich, B. Sudakov, and T. Szabó, Triangle factors in sparse pseudo-random graphs, Combinatorica 24 (2004), no. 3, 403-426. MR2085364 (2005g:05120) $\uparrow 2,3$

[36] D. Kühn and D. Osthus, On Pósa's conjecture for random graphs, SIAM J. Discrete Math. 26 (2012), no. 3, 1440-1457. $\uparrow 4$

[37] M. Kwan, Almost all Steiner triple systems have perfect matchings (2016), available at arXiv:1611.02246. $\uparrow 9$

[38] A. Lubotzky, R. Phillips, and P. Sarnak, Ramanujan graphs., Combinatorica 8 (1988), no. 3, 261-277. $\uparrow 2,9$

[39] S. Micali and V. V. Vazirani, An $O\left(|V|^{1 / 2}|E|\right)$ algorithm for finding maximum matching in general graphs, Proceedings of the 21st Annual Symposium on Foundations of Computer Science, 1980, pp. 17-27. $\uparrow 11,12$

[40] R. H. Montgomery, Embedding bounded degree spanning trees in random graphs (2014), available at arXiv:1405.6559v2. $\uparrow 4,9$

[41] R. Nenadov, Triangle-factors in pseudorandom graphs, Bulletin of the London Mathematical Society, to appear (2018). $\uparrow 2,3,4,5,17,18$

[42] R. Nenadov and Y. Pehova, On a Ramsey-Turán variant of the Hajnal-Szemerédi theorem, arXiv preprint arXiv:1806.03530 (2018). $\uparrow 9$

[43] V. Rödl, A. Ruciński, and E. Szemerédi, A Dirac-type theorem for 3-uniform hypergraphs, Combin. Probab. Comput. 15 (2006), no. 1-2, 229-251. $\uparrow 4$

[44] — An approximate Dirac-type theorem for k-uniform hypergraphs, Combinatorica 28 (2008), no. 2, 229-260. $\uparrow 4$ 
[45] A. Thomason, Pseudorandom graphs, Random graphs '85 (Poznań, 1985), 1987, pp. 307-331. MR930498 ^1

[46] _ Random graphs, strongly regular graphs and pseudorandom graphs, Surveys in combinatorics 1987 (New Cross, 1987), 1987, pp. 173-195. MR905280 ^1

[47] A. Walfisz, Zur additiven Zahlentheorie. II., Mathematische Zeitschrift 40 (1936Dec), no. 1, 592-607. $\uparrow 10$

Department of Mathematics, University of Rhode Island, 5 Lippitt Road, Kingston, RI, USA, 02881

E-mail address: jie_han@uri.edu

Instituto de Matemática e Estatística, Universidade de São Paulo, Rua do Matão 1010, 05508-090 SÃo Paulo, Brazil

E-mail address: yoshi@ime.usp.br

Institut für Mathematik, Freie Universität Berlin, Arnimallee 3, 14195 Berlin, Germany And Berlin Mathematical School, Germany

E-mail address: pm0041@mi.fu-berlin.de

Institut für Mathematik, Technische Universität Ilmenau, 98684 Ilmenau, Germany

E-mail address: yury.person@tu-ilmenau.de 